\title{
Aspecto histopatológico do Carcinoma Adenoide Cístico em cavidade bucal: relato de caso
}

\author{
Histopathological aspects of Cystic Adenoid Carcinoma: case report \\ Aspectos histopatológicos del Carcinoma Adenoide Quístico en cavidad oral: reporte de \\ caso
}

Camilla Lins dos Anjos ${ }^{1 *}$, Laís Regina de Oliveira Cavalcanti ${ }^{1}$, Karla Alves Chagas ${ }^{1}$, Mycaelle Stephanny das Neves Barbosa Sena ${ }^{1}$, Fernanda Braga Peixoto ${ }^{1}$, Sonia Maria Soares Ferreira ${ }^{1}$, Camila Maria Beder Ribeiro Girish Panjwani¹.

\section{RESUMO}

Objetivo: Relatar o caso clínico de Carcinoma Adenóide Cístico (CAC), mostrando características clínicas, histopatológicas e radiográficas da lesão. Detalhamento do caso: Paciente sexo feminino, 41 anos, compareceu ao serviço, com a queixa principal "vim por inchaço no dente". Na história da doença atual, a paciente relatou que a lesão surgiu há aproximadamente dois anos, no palato duro, a lesão começou pequena, cresceu lentamente e no início apresentava dor. Ao exame intraoral, foi detectado, lesão nodular extensa. A hipótese diagnóstica foi de Adenoma Pleomórfico. Realizou-se biópsia incisional e foi diagnosticada com CAC de padrão histológico misto: sólido e cribriforme, sendo encaminhada ao serviço oncológico, onde a paciente segue em tratamento quimioterápico e radioterápico Considerações finais: CAC é um tumor maligno raro e incomum de glândulas salivares menores. Em geral a excisão cirúrgica é o tratamento de escolha e, a radioterapia adjuvante, pode melhorar a sobrevida dos pacientes. Vários fatores são considerados para o prognóstico do CAC, dentre eles o tipo histológico e estágio clínico da doença. Destaca-se a importância da realização de exames complementares para o correto diagnóstico.

Palavras-chave: Carcinoma, Diagnóstico, Etiologia.

\begin{abstract}
Objective: Is to report the case of Adenoid Cystic Carcinoma (ACC), showing clinical characteristics, histopathological and radiographic of the injury. Case detailing: Patient female, 41 years old, attended the service, with the main complaint "I came because of tooth swelling". In the history of the current disease, the patient reported that the lesion appeared approximately two years ago, in the hard palate, the lesion started small, grew slowly and at first presented pain. An intraoral examination revealed an extensive nodular lesion. The hypothesis diagnosed was Pleomorphic Adenoma. An incisional biopsy was performed and was diagnosed with mixed histological pattern: solid and cribriform ACC, being referred to the cancer service, where the patient is undergoing chemotherapy and radiotherapy. Final considerations: ACC is a rare and uncommon malignant tumor of the minor salivary glands. Surgical excision is generally the treatment of choice, and adjuvant radiotherapy can improve patient survival. Several factors are considered for the prognosis of ACC, including the histological type and clinical stage of the disease. This study highlights the importance of performing complementary exams for the correct diagnosis.
\end{abstract}

Key words: Carcinoma, Diagnosis, Etiology.

${ }^{1}$ Centro Universitário Cesmac, Maceió-Alagoas. *E-mail: camillanjos@gmail.com 


\section{RESUMEN}

Objetivo: Relatar un caso clínico de Carcinoma Adenoideo Quístico (CAQ), mostrando características clínicas, histopatológicas y radiográficas de la lesión. Reporte del caso: paciente del género femenino, 41 años, acudió al servicio con queja principal "vine porque tengo hinchazón en el diente". En la historia actual de la enfermedad, la paciente ha relatado que la lesión surgió desde hace aproximadamente 2 años, en el paladar duro. La lesión empezó pequeña, creció lentamente y al principio presentaba dolor. En el examen intraoral fue detectada una lesión nodular extensa. La hipótesis de diagnóstico fue de Adenoma Pleomorfo. Se realizó biopsia incisional y fue diagnosticada con $\mathrm{CAQ}$ de dos patrones histológico: sólido y cribiforme, por lo tanto ha sido encaminada al servicio oncológico, donde la paciente sigue con el tratamiento de quimioterapia y radioterapia. Consideraciones finales: CAQ es un tumor maligno raro e inusual de glándulas salivales menores. Generalmente, la extirpación quirúrgica es el tratamiento elegido y, la radioterapia adyuvante, puede mejorar la sobrevida de los pacientes. Se consideran varios factores para el pronóstico del $C A Q$, entre ellos el tipo histológico y estadio clínico de la enfermedad. Se resalta la importancia de realizar exámenes complementares para un diagnóstico correcto.

Palabras claves: Carcinoma, Diagnóstico, Etiología.

\section{INTRODUÇÃO}

As neoplasias das glândulas salivares acometem adultos entre 50 a 70 anos de idade, há uma distribuição igual entre os sexos, embora alguns estudos mostrem prevalência no sexo feminino (NEVILLE BW, et al., 2016; SANTOS T, et al., 2011; TINOCO P, et al., 2009).

O Carcinoma Adenoide Cístico (CAC) é um tumor maligno raro e incomum de glândulas salivares menores, que corresponde de 3 a $5 \%$ de todos os tumores malignos da cabeça e pescoço (DANTAS AN, et al., 2015; GIÃO M, et al., 2017; SANTOS T, et al., 2011).

A fisiopatologia do CAC ainda é desconhecida, embora considere-se que tenha origem nas células epiteliais das glândulas secretoras de muco, especificamente nas glândulas menor subepiteliais (MONTEIRO D, et al., 2012). Mesmo com o controle local da doença ele é conhecido por ter apresentação metastática tardia, com mais frequência a nível pulmonar (TINOCO P, et al., 2009).

A otalgia é o principal sintoma inicial em $90 \%$ dos pacientes, antes que ocorra tumefação. Apresenta-se clinicamente com um aumento de volume, de crescimento lento, consistência endurecida semelhante a um nódulo, recoberto por mucosa íntegra. Os pacientes geralmente queixam-se de uma dor específica, constante e de baixo grau, que aumentam de intensidade gradativamente. Apesar de rara, a ulceração pode ser observada nas lesões localizadas no palato e, ao exame radiográfico exibe aspecto de destruição óssea. Os tumores que se originam no palato ou no seio maxilar geralmente exibem características de destruição óssea (NEVILLE BW, et al., 2016; SANTOS T, et al., 2011; TINOCO P, et al., 2009).

Histologicamente, o CAC é composto por uma mistura de células mioepiteliais e ductais com formas variáveis, podendo ser reconhecidos três padrões principais: cribiforme, tubular ou sólido (NEVILLE BW, et al., 2016; SARMENTO DJS, et al., 2010).

Cribiforme é o padrão mais comum, apresenta ilhas de células basolóides que contém múltiplos espaços cilíndricos, semelhantes a espaços císticos; (NEVILLE BW, et al., 2016). No tubular, as células epiteliais estão arranjadas em vários ductos, envolvidos no estroma hialino e na variante sólida, ilhas ou lençóis que demonstram pouca tendência a formar ductos ou espaços císticos (WELLING LC, et al., 2011).

Já no sólido apresenta-se grandes ilhas ou ninhos pálidos de células tumorais que demonstram pouca tendência para formação de ductos ou cistos, diferente dos dois outros padrões pode observar pleomorfismo celular e atividade mitótica bem como focos de necrose no centro das ilhas tumorais (NEVILLE BW, et al., 2016). A invasão perineural consiste em um achado clínico, comum de dor nesses pacientes óssea (NEVILLE BW, et al., 2016). 
O tratamento de escolha é a excisão cirúrgica, combinado ou não com a radioterapia, embora não tenha sido comprovada categoricamente a sua eficácia (NEVILLE BW, et al., 2016).

Os fatores de prognósticos mais importantes são: o estágio inicial (TNM); o tipo histológico, a sua localização e a persistência tumoral pós-cirurgia. Em princípio o prognostico mantém-se guardado com sobrevida de 50\% em cinco anos (WELLING LC, et al., 2011).

Os tumores com padrão histolopatológico solido estão associados a um prognostico pior do que o observado para os padrões cribriforme ou tubular. De acordo com a localização, o prognostico é pior para tumores originários do seio maxilar e da glândula submandibular (NEVILLE BW, et al., 2016).

O objetivo desse trabalho é demonstrar a importância dos exames complementares para um correto diagnóstico das lesões orais.

\section{RELATO DE CASO}

Paciente feminina, 41 anos, melanoderma, procurou o serviço de estomatologia do Centro Universitário CESMAC, com a queixa principal "vim por inchaço no dente", segundo ela a lesão surgiu há aproximadamente 2 anos, no palato duro, percebeu ao tentar colocar prótese; relata crescimento lento e dor apenas inicialmente.

No exame extrabucal não teve presença de linfonodos palpáveis. Na avaliação intrabucal foi observada

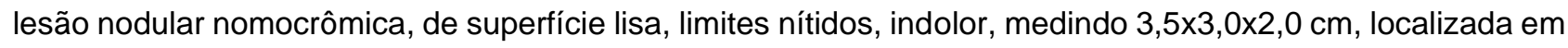
palato duro (Figura 1).

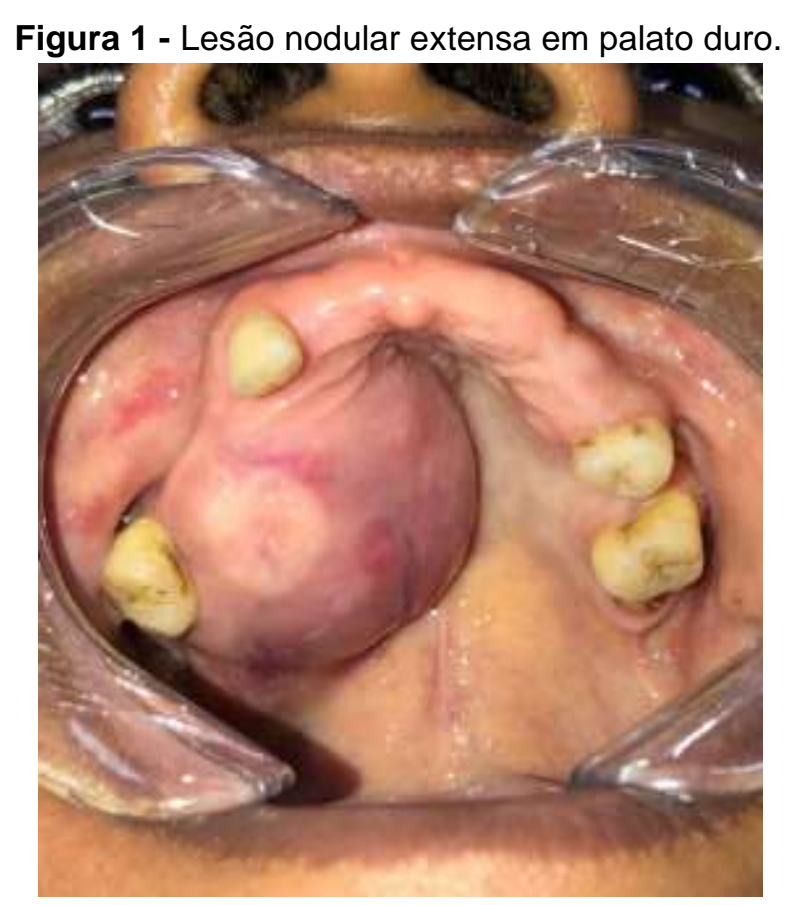

Fonte: Anjos CL, et al., 2019.

As principais hipóteses diagnósticas foram: adenoma pleomórfico, adenocarcinoma. Foi realizada biópsia incisional e o fragmento da lesão foi encaminhado para o laboratório de patologia bucal do Centro universitário CESMAC.

No exame histológico revelaram fragmentos de neoplasia maligna de origem epitelial glandular. As células neoplásicas apresentam padrão basoloide e estão dispostas em ilhas que ora contém múltiplos espaços císticos ora apresentam com padrão mais cribriforme destruídas em um estroma fibrovascular e hemorrágico, assim como também apresenta padrão sólido (Figura 2 e 3). 
Figura 2 - Fotomicroscopia da lesão biopsiada, evidenciando padrão histológico cribriforme $(\rightarrow$ ) (corada em HE no aumento de 400x).

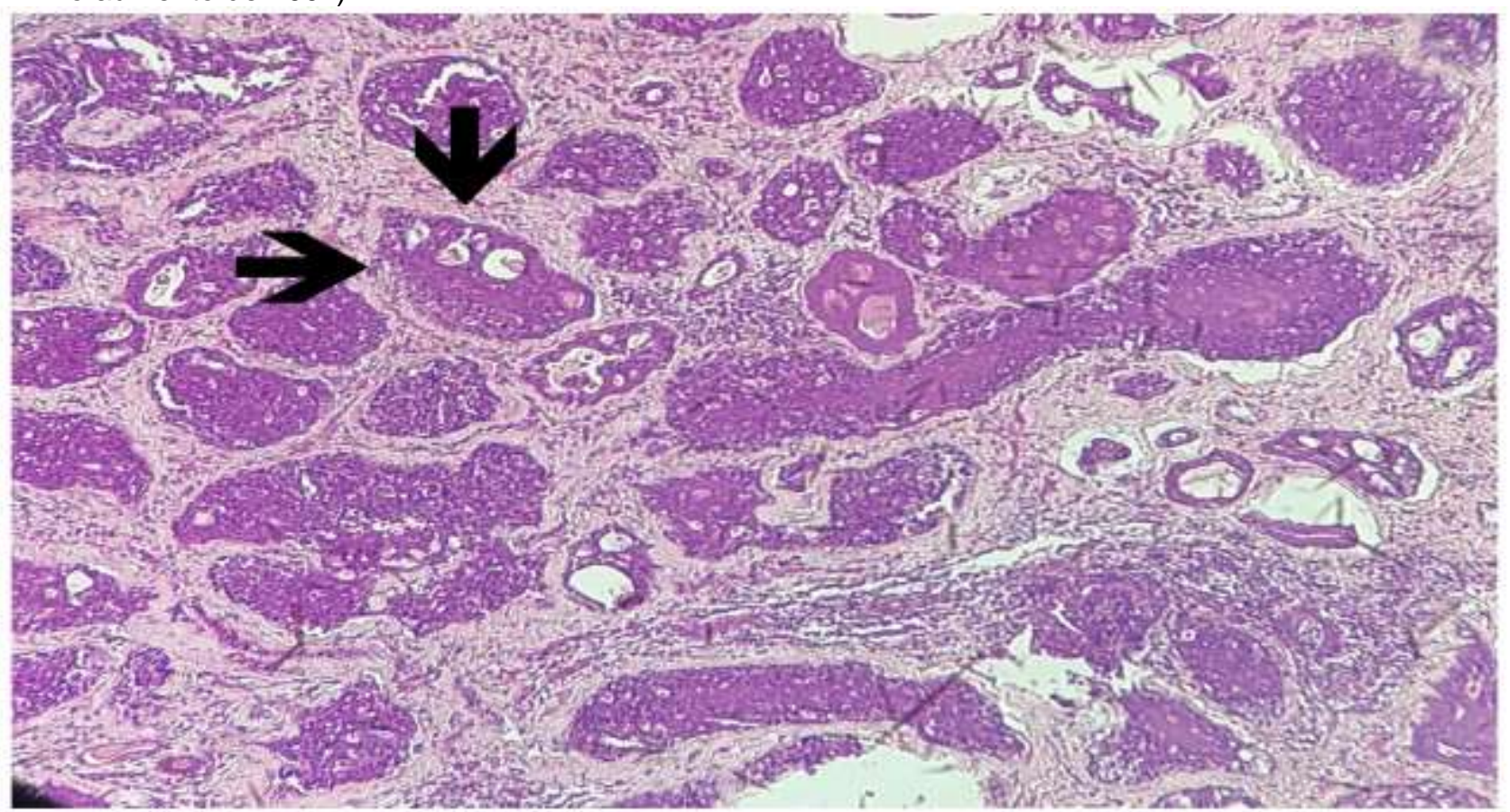

Fonte: Anjos CL, et al., 2019.

Figura 3 - Fotomicroscopia da lesão biopsiada, evidenciando predominância de padrão sólido $(\rightarrow)$ (corada em HE no aumento de 400x).

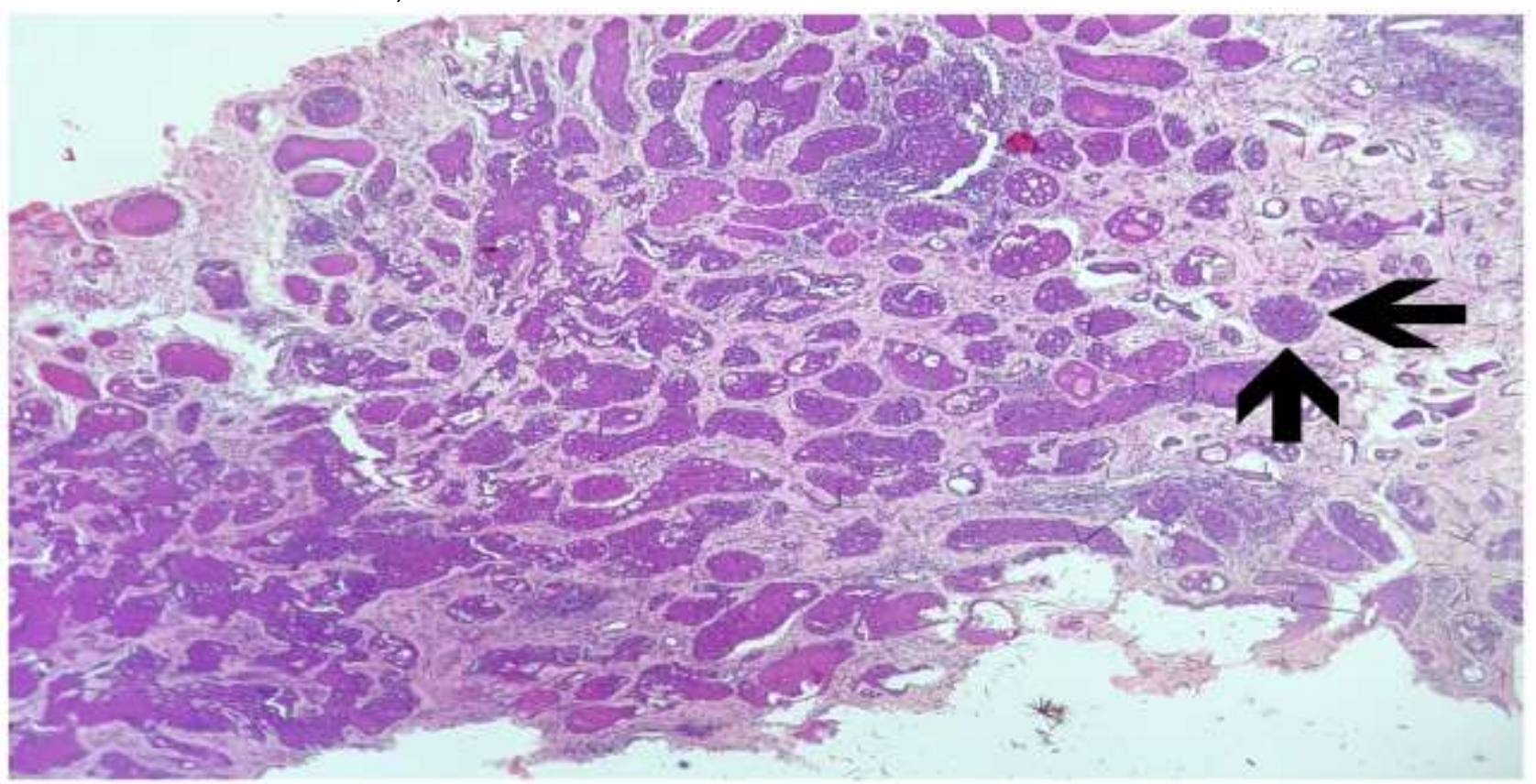

Fonte: Anjos CL, et al., 2019.

Após laudo histopatológico a paciente foi encaminhada ao Centro Oncológico, antecipando-se a exames que poderiam ser solicitados, como tomografia computadorizada. Destaca-se: massa tumoral na maxila do lado direito, suavemente delimitada e invasiva, com expansão, adelgaçamento e extensa destruição óssea nas regiões de palato duro, osso alveolar, que se estende para a região de seio maxilar direito, fossa nasal, seio etmoidal, seio esfenoidal e alcança o soalho da órbita (Figura 4 e 5). 
Figuras 4 e 5 - Tomografia computadorizada de feixe cônico e corte axial.

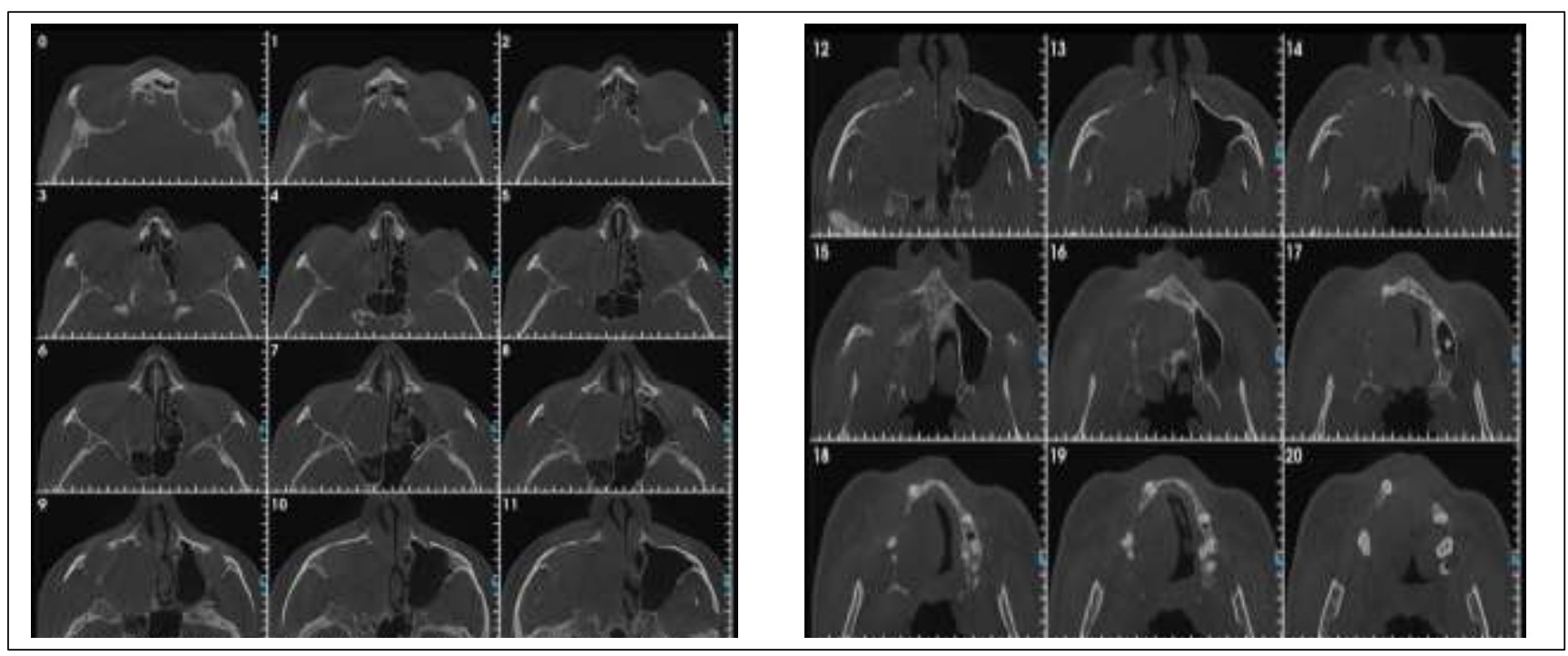

Fonte: Anjos CL, et al., 2019.

Após os exames complementares, a paciente foi encaminhada para serviço oncológico, a qual, está submetida ao tratamento radioterápico e quimioterápico.

\section{DISCUSSÃO}

De acordo com a literatura escolhida durante a análise sistemática, observaram alguns fatores do o CAC localizados em cabeça e pescoço, a lesão afeta ambos os sexos, porém tem maior predileção pelo gênero feminino (DANTAS AN, et al., 2015, NEVILLE BW, et al., 2016). No caso relatado constata-se lesão em paciente do sexo feminino, 41 anos de idade. Alguns autores relatam que a faixa etária mais constantemente afetada é entre a $5^{\underline{a}}$ a $7^{\text {a }}$ décadas de vida (GIÃO M, et al., 2017). No caso apresentado a paciente era de faixa etária adulta de vida, no qual difere dos casos relatados na literatura.

Alguns achados literários (SANTOS T, et al., 2011; BW, et al., 2016; SARMENTO DJS, et al., 2010), relatam que a lesão do CAC se origina em glândulas salivares menores (aproximadamente $50 \%$ a $60 \%$ dos casos), sendo o principal sítio intraoral acometido o palato. Em geral o principal sintoma inicial é a dor. No caso relatado a lesão surgiu no palato duro com aproximadamente 2 anos de evolução, com crescimento lento e com sintomatologia dolorosa no início.

O CAC normalmente apresenta-se com crescimento lento, consistência endurecida, recoberto por mucosa íntegra e é raro haver ulceração (NEVES FS, et al., 2010; SANTOS T, et al., 2011; SARMENTO DJS, et al., 2010). Neste caso a lesão encontrava-se com superfície lisa, consistência endurecida, sem ulceração, o que é condizente com a literatura.

Os casos de recidiva da lesão são frequentemente encontrados no CAC, no qual está diretamente associado à sua localização, em glândulas salivares menores. Esta associação está diretamente ligada aos casos com maior agressividade da invasão tumoral e prognóstico desfavorável. A frequência de recidiva local do carcinoma adenoide cístico é comum, demandando novas ressecções cirúrgicas. No caso descrito, a lesão da paciente está localizada em glândulas salivares menores e com o caso mais avançado (DANTAS AN, et al., 2015; NEVILLE BW, et al., 2016).

O diagnóstico histopatológico do CAC requer uma biópsia incisional adequada, no entanto, autores afirmam que o CAC é composto por uma mistura de células mioepiteliais e ductais com formas variáveis que podem ser reconhecidas como três padrões principais cribiforme, tubular ou sólido (NEVILLE BW, et al., 2016; SARMENTO DJS, et al., 2010). No presente caso, o tumor é de neoplasia maligna de origem epitelial glanduar e histologicamente misto, padrão cribiforme e sólido o que é concordante com tumores de glândulas salivares menores em palato descritos por esses autores. 
Outras hipóteses de diagnóstico diferencial também podem ser propostas dentre as demais lesões de glândula salivar, tais como neoplasias benignas (adenoma pleomórfico) e malignas (adenocarcinoma polimorfo de baixo grau e carcinoma mucoepidermoide) (SARMENTO DJS, et al., 2010). Quando é efetuado antecipadamente o diagnóstico do carcinoma adenoide cístico, o paciente é capaz de possuir uma melhor condição de vida e aumento de sobrevida. No caso da paciente, já havia 2 anos de crescimento quando foi descoberto.

É comum encontrar como um achado histológico a invasão perineural, podendo ser vista uma provável via de difusão das células tumorais. Quando localizadas em região de glândulas salivares menores facilita o reaparecimento e pode estar relacionado com casos mais evoluídos e com uma estimativa ruim, podendo comprometer grande margem de incidência cirúrgica. A abrangência perineural, ocorre na maior parte dos casos de CAC, encontrando-se em padrão micro ou macroscopicamente (DANTAS AN, et al.,2015). No caso relatado, não foi apresentado invasão perineural.

Diversos autores observaram o aparecimento de metástase a distância nos pacientes acometidos com o $C A C$, estando especialmente o pulmão o local mais frequentemente atingido, junto com cérebro, osso, fígado e outros sitios. Sendo capaz de aparecer depois da ressecção primária. A literatura afirma, que a dissecção linfática da cervical deve ser exclusiva nos acometidos em que se encontra em manifestação clínica de metástase no pescoço (ALVES AT, et al., 2014). O que não foi notado neste paciente durante os procedimentos realizados.

O tratamento de escolha foi a excisão cirúrgica, antecedendo-se de exames propostos pelo centro oncológico, seguido por radioterapia. De acordo com os autores o tratamento do CAC de palato é a excisão cirúrgica local englobando sua mucosa de recobrimento, incluindo o tecido normal, com o objetivo de se evitar recidivas, seguido ou não de radioterapia e em raros casos, quimioterapia. O esvaziamento cervical, como forma de tratamento é utilizado apenas para casos em que se tem em vista baixo índice de comprometimento metastático nodal, analisados no exame clínico ou imaginológico (NEVILLE BW, et al., 2016; TINOCO P, et al., 2009).

\section{CONSIDERAÇÕES FINAIS}

O CAC é um tumor maligno de crescimento lento, poder ser dolorido no início e assintomático depois de um determinado tempo. As características clínicas podem ser confundidas com outras patologias, sendo indispensável a realização de exame histopatológico para o correto diagnóstico. O tratamento de escolha é o cirúrgico conservador com alto índice de recidiva e probabilidade de metástase a distância.

\section{REFERÊNCIAS}

1. DANTAS AN, et al. Clinicopathological characteristics and perineural invasion in adenoid cystic carcinoma: a systematic review. Braz J Otorrinolaringol, 2015; 81(3):329-335.

2. ALVES AT, et al. Adenoid cystic carcinoma: review of the literature and case report. $2014 ; 40(6): 421-424$.

3. BERNARDES VF, et al. Carcinoma adenóide cístico sólido em palato e seio maxilar. Rev. Bras. Otorrinolaringol, 2006; 72(4): 573-573.

4. GIÃO M, et al. Carcinoma Adenoide Quístico das fossas nasais e seios perinasais- Revisão retrospetiva de 20 anos. revista portuguesa de otorrinolaringologia e cirurgia cérvico-facia, 2017; 55(3): 163-166.

5. MONTEIRO D, et al. Carcinoma adenóide cístico laríngeo: A propósito de um casoclínico. Revista portuguesa de otorrinolaringologia e cirurgia cérvico-facial, 2012; 50(1): 65-69.

6. NEVES FS, et al. Carcinoma Adenoide Cístico em uma Localização Incomun. Cir.Traumatol. Buco- Maxilo-Fac, 2010; 10(4):17-20.

7. NEVILLE BW et al. Patologia oral e maxilo facial. Rio de Janeiro: Elsevier, 2016; 928p.

8. SANTOS T, et al. Adenoid cystic carcinoma of mandible. Brazilian Journal of Otorhinolaryngology, $2011 ; 77(6): 807$.

9. SARMENTO DJS, et al. Diagnóstico e conduta do cirurgião-dentista frente ao carcinoma adenóide cístico. Passo Fundo, 2010; 15(2): 203-206.

10. TINOCO P, et al. Carcinoma Adenoide Cístico do Conduto Auditivo Externo com Envolvimento de Mastoide. Int. Arch. Otorrinolaringol, 2009;13(2): 221-225.

11.WELLING LC, et al. Carcinoma adenoide cístico intracraniano. Arq Bras Neurocir, 2011. 30(2): 39-41. 